Campbelu, L. L. Jun. \& Williams, O. B. (1951). J. gen. Microbiol. 5, 894-905.

\title{
A Study of Chitin-decomposing Micro-organisms of Marine Origin
}

\author{
By L. L. CAMPBELL, Jun. AND O. B. WILLIAMS \\ Department of Bacteriology, University of Texas, Austin, Texas, U.S.A.
}

SUMMARY: Twenty strains of aerobic, facultatively anaerobic, chitinoclastic bacteria have been isolated from marine mud by enrichment cultures. Each was able to derive its full carbon and nitrogen requirements from chitin. None was an obligate chitinovor.

These cultures comprised four new species of Achromobacter, two new species of Pseudomonas, one new species of Flavobacterium and one new species of Micrococcus. Detailed descriptions for each species are appended.

Each organism was able to liberate ammonia and reducing sugar from the chitin molecule. Glucosamine and acetic acid were not detected in the cultures, possibly because of their ready availability as supplementary nutrients.

Chitin is a tough leathery substance insoluble in the usual organic solvents, requiring strong mineral acid for its solution. It is a nitrogen-containing polysaccharide, generally believed to be composed of polymerized $N$-acetylglucosamine united by beta-1, 4 linkages to form long chains which are orientated in much the same manner as the glucose units in cellulose.

Chitin has a wide distribution in nature. It occurs in the exo-skeleton of crustacea, in some mollusca, coelenterata, protozoa (ZoBell, 1946) and in the cell wall of certain lower fungi and filamentous yeast (Nabel, 1939). Waksman (1933) has reported that it occurs as one of the nitrogenous constituents of marine sediments.

It has been estimated that several million tons of chitin are produced annually by the copepoda, a subclass of planktonic crustacea. This, as well as the chitin produced by other organisms in the sea, is probably utilized by biological agents because little accumulates in marine sediments, and, moreover, if the chitin were not decomposed its accumulation would soon cause a serious drain on the marine carbon and nitrogen (ZoBell \& Rittenberg, 1938). There are few animals which can attack chitin, and it is generally agreed that its degradation in nature is largely due to microbial action.

Since Benecke (1905) reported the isolation of Bacillus chitinovorus from the polluted waters of Kiel harbour, there have appeared numerous reports of the isolation of micro-organisms capable of decomposing chitin. The reports of Steiner (1931), Johnson (1932), Waksman, Carey \& Reuszer (1933), ZoBell \& Anderson (1936), ZoBell \& Rittenberg (1938), Hock (1941), Erikson (1941) and Stanier (1947) on the occurrence of chitin-decomposing micro-organisms from marine sources, together with the report of Stuart (1936) on the occurrence of halophilic chitinovorous bacteria in marine salt from various parts of the world, reveals the world-wide distribution of chitin-decomposing micro-organisms in the seas. Chitinovors have been isolated from terrestrial sources by Folpmers 
(1921), Rammelberg (1931), Johnson (1932), Bucherer (1935), Benton (1935) and Skinner \& Dravis (1937).

ZoBell \& Rittenberg (1938), by the use of enrichment culture methods, isolated thirty-one different types of chitinoclasts and found that they differed greatly in their nitrogen and carbon (energy) requirements as well as their action on chitin. Chitinoclastic activity took place more readily aerobically than anaerobically. Many of their chitinoclasts produced acetic acid and ammonia, suggesting to them that certain chitinoclasts attack the chitin molecule by hydrolysis of the acetylated amino groups. Others were able to attack the essential carbon and oxygen linkages of the chitin molecule resulting in a complete disintegration and the liberation of reducing sugars.

Few of the previous reports on chitinoclastic bacteria have given enough information to justify an opinion as to whether the organisms isolated were representatives of known genera and species or not. The investigation here reported was undertaken to obtain some information on this point.

\section{METHODS AND MATERIALS}

Chitin was prepared from the exoskeleton of shrimp by the method of Benton (1935). The material was thoroughly washed in warm water and decalcified in cold $1 \%(\mathrm{v} / \mathrm{v}) \mathrm{HCl}$, which was changed every day for 4 days. It was then washed, placed in $2 \%(\mathrm{w} / \mathrm{v}) \mathbf{K O H}$ for about 10 days, and on four separate occasions brought to a boil and allowed to cool. It was then washed free from alkali, cut in strips and extracted repeatedly with boiling ethanol and dried. The purified strips gave positive tests for chitin as listed by Dehn (1937), and by Glick (1949).

Enrichment cultures for chitin-decomposing micro-organisms were made by adding strips of purified chitin to a thin layer of dilute mineral base $\left(\mathrm{K}_{2} \mathrm{HPO}_{4}\right.$ $0.1 \%$ (w/v), $\mathrm{MgSO}_{4} 0.05 \%$ (w/v), $\mathrm{NaCl} 0.05 \%$ (w/v), $\mathrm{CaCl}_{2} 0.01 \%$ (w/v), $\mathrm{FePO}_{4}$ trace) in flat glass bottles. A second series of enrichments was set up in the same manner, but with the addition of $0 \cdot 1 \%(\mathrm{w} / \mathrm{v}) \mathrm{NH}_{4} \mathrm{Cl}$ to the mineral base. The media were adjusted to $\mathrm{pH} \mathbf{7 \cdot 0}$, inoculated with marine mud and incubated at $25-30^{\circ}$.

For chitin agar plates the purified strips were dissolved in $50 \%(\mathrm{v} / \mathrm{v}) \mathrm{H}_{2} \mathrm{SO}_{4}$ and precipitated by rapid dilution with a 15 -fold volume of water. The resulting finely divided chitin was freed from acid by washing on a Buchner funnel until the filtrate was neutral to litmus. This wet material was incorporated into the mineral base agar directly, as drying results in the formation of a hard mass which is impossible to resuspend.

Following serial enrichment cultures, streaks were made on the chitin agar plates. These were examined daily for the appearance of haloes, indicative of chitin decomposition, surrounding the colonies. Colonies showing haloes were isolated, purified and finally transplanted to nutrient agar slants for further study.

For studies on chitin decomposition, flat glass bottles containing thin layers of the dilute mineral base $(\mathrm{pH} 7 \cdot 0)$ and strips of purified chitin were inoculated 
with pure cultures of the chitin-decomposing organisms. These were examined, at $2,4,6,8,12,16$ and 20 days, for ammonia, acetate, glucosamine, reducing sugars and for changes in $\mathrm{pH}$. Changes in $\mathrm{pH}$ were measured electrometrically. Ammonia was determined with Nessler's reagent; glucosamine by the method of Elson \& Morgan (1933), and reducing sugars with Barfoed's and Fehling's reagents.

The ability to use various carbon compounds was tested by adding $1 \%(\mathrm{w} / \mathrm{v})$ (unless otherwise noted) of the compound to the mineral base with $0 \cdot 1 \%(\mathrm{w} / \mathrm{v})$ $\mathrm{NH}_{4} \mathrm{Cl}$, and observing for growth. Media for the study of other biochemical characters were prepared in the usual manner. The arrangement of the flagella of motile forms was determined by both Zettnow staining and electron microscope examination.

\section{RESULTS}

Chitin decomposition in enrichment cultures was manifested by erosion of the strips of chitin after 8-12 days at 25-30 ${ }^{\circ}$. Gram-stained preparations from the enrichment cultures revealed the presence of a flora consisting of small rods and cocci. Streaks from enrichment cultures on chitin-mineral agar revealed, in 2-3 days, several colonial types surrounded by clear haloes in which the chitin had been dissolved.

Twenty colonies were picked and purified; from further study these appeared to include eight different species of chitin-decomposing organisms. All of the strains isolated were true bacteria and were distributed among the genera Achromobacter, Pseudomonas, Flavobacterium and Micrococcus. The failure to detect any of the myxobacteria types is probably due to the fact that they grow in an inconspicuous manner and at a slow rate, and thus may be easily overlooked (Stanier, 1947).

The eight species reported here have been studied in detail in regard to chitin decomposition, carbon utilization, and by various other morphological, biochemical and physiological tests. The positive results obtained from the study of chitin decomposition are summarized in Table 1 . The results of the other tests are included as a part of the description of each organism. Species descriptions with the names which have been selected are appended to this report. All tests for acetate and glucosamine were negative. All cultures became acid after from 4 to 8 days. The $\mathrm{pH}$ range was from $5 \cdot 5$ to $6 \cdot 0$.

\section{DISCUSSION}

The organisms isolated in the present study were all representatives of wellknown genera for which species descriptions seem not to have been recorded previously. Because of the incomplete descriptions of the aerobic bacteria with chitinoclastic ability which have been isolated by the few earlier workers who have studied such organisms it is not possible to identify any of the types here reported with previous isolates.

Probably the most extensive study of chitinoclastic bacteria of marine origin is that of ZoBell \& Rittenberg (1938). Some of their isolates were able to grow on a medium consisting of purified chitin in sea water, others grew only if supplied with a supplementary source of carbon, and still others required that 


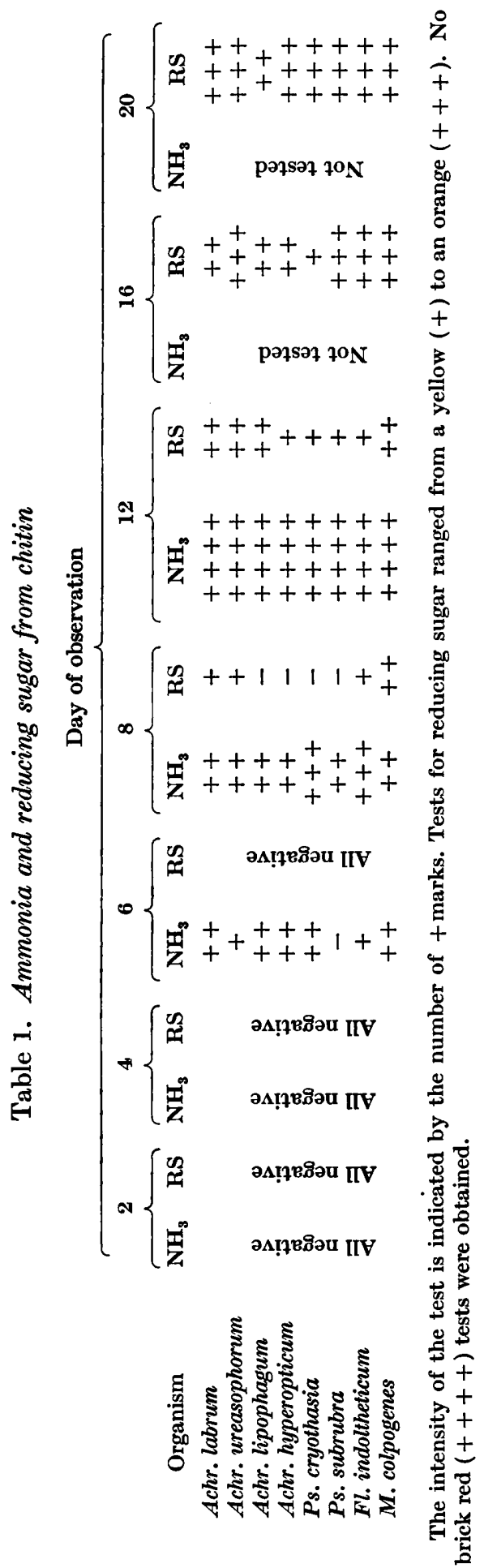


additional sources of both carbon and nitrogen be supplied. Despite the fact that chitin is presumably a polymerized glucosamine some of their cultures were unable to attack glucose, although able to effect the hydrolysis of starch. Some ZoBell \& Rittenberg types grew without detectable effect on the chitin while others would dissolve it. They postulated that the chitin molecule is attacked either by hydrolysing the acetylated amino groups with the liberation first of acetic acid and then of ammonia, or by the hydrolysis of the carbonnitrogen linkage with the liberation of acetamide from which ammonia and acetic acid were freed by further hydrolytic activity. They were able to detect acetic acid in some of their cultures. Most of their organisms were able to use the ammonium ion, but ammonia must have been produced in excess of the metabolic needs since apparently all cultures showed a positive test for ammonia. Not all types produced reducing sugar, and not all cultures developed acidity.

All organisms isolated in the present study were able to use ammonia as a source of nitrogen. All except one produced enough ammonia to show a positive test by the sixth day. One culture, Pseudomonas subrubra, was generally less vigorous than any of the others. It was not positive for ammonia until the eighth day, and for acid only by the twelfth day, although the other cultures were acid in from 4 to 8 days.

A positive test for acetate was not obtained with any culture at any time. If the theory of ZoBell \& Rittenberg concerning the mechanism of chitin breakdown is correct, the failure to detect acetate may possibly be explained by the more ready availability and consequently preferential utilization of the acetate carbon to such an extent that it does not accumulate sufficiently in the culture to give a positive test. This theory finds support in the fact that maximum growth was more quickly attained in cultures where acetate was supplied as a supplementary source of carbon than where chitin was the sole carbon source.

The breakdown of the chitin molecule into glucosamine units is a possibility, but all tests for glucosamine were negative. Reducing sugar appeared in some cultures by the eighth day and in all by the twelfth day, although the positive reactions were not as intense as that of the glucose control. Since all cultures were able to utilize glucosamine it could be that this substance was preferentially metabolized as was suggested for acetate. All cultures produced acid from chitin in from 4 to 12 days.

Five of the eight types studied were proteolytic and four were lipolytic. All except two were weakly saccharolytic, producing acid without gas from the sugars which were attacked.

The authors are indebted to Dr W. J. Battle for his invaluable assistance in selection of the species names. The work upon which this report is based was supported in part by a research grant from the National Institutes of Health, Public Health Service, U.S.A. 


\section{CHARAGTERISTICS OF ISOLATED STRAINS}

Achromobacter labrum n.sp. (from the Greek, greedy)

Rods : $0 \cdot 2-0 \cdot 4$ by $0 \cdot 8-1 \cdot 1 \mu$, occuring singly, in pairs and occasionally in clumps. Motile by means of peritrichous flagella. Gram-negative.

Agar colonies : circular, smooth, entire, flat, glistening, opaque, non-pigmented to pale cream.

Agar slant: abundant filiform streak, smooth, glistening, opaque, nonpigmented.

Gelatin stab: no liquefaction.

Broth: moderate turbidity; surface growth adherent to the walls of the tubes.

Litmus milk: becomes acid in 6 days.

Hydrogen sulphide, indole and urease not formed.

Nitrites produced from nitrates.

Trimethylamine not produced from trimethylamine oxide, choline or betaine. Gelatin, casein, and fats not hydrolysed; starch and chitin are hydrolysed.

Ammonia produced from peptone.

Produces acid but no gas from glucose, maltose, fructose, dextrin, mannose, trehalose, cellobiose. Acid not produced from lactose, sucrose, arabinose, rhamnose, xylose, raffinose, inulin, mannitol, salicin, dulcitol, inositol, melezitose, adonitol.

Carbon utilization: grows on glucose, lactose, sucrose, maltose, fructose, dextrin, mannose, arabinose, rhamnose, xylose, raffinose, inulin, mannitol, salicin, galactose, trehalose, inositol, cellobiose, melezitose, adonitol, chitin, glycogen, starch, butyrate, valerate, asparaginate, succinate, malate, fumarate $(0.5 \%)$, lactate, acetate, tartrate, citrate, pyruvate $(0.3 \%)$, levulinate, $\beta$-alanine, glucosamine, ethanol and tert.-butanol. No growth on dulcitol, cellulose, malonate, salicylate, oxalate, mandelate, benzoate $(0.5 \%)$, propionate, $n$-amyl alcohol, methanol, $n$-propanol, iso-amyl alcohol, lumichrome.

Growth not inhibited by $10 \%$ sodium chloride.

Aerobic, facultative.

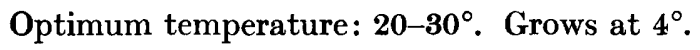

Source: marine mud.

Habitat: sea water.

One additional strain.

Achromobacter ureasophorum n.sp. (from the Greek, urease producing)

Small rods: $0 \cdot 2$ by $0 \cdot 8 \mu$., occurring singly, motile by means of peritrichous flagella. Gram-negative.

Agar colonies: circular, raised, glistening, entire, translucent, non-pigmented. Agar slant: abundant, filiform, glistening, translucent, non-pigmented.

Gelatin stab: no liquefaction.

Broth: slight turbidity, with a stringy sediment.

Litmus milk: becomes slightly acid in 6 days.

Hydrogen sulphide, indole, nitrites not produced. 
Urease present.

Trimethylamine not produced from trimethylamine oxide, choline, or betaine. Gelatin, casein and fats not hydrolysed; starch and chitin are hydrolysed.

Ammonia produced from peptone.

Produces acid but not gas from glucose, maltose, sucrose, fructose, mannose, trehalose, and cellobiose. Does not ferment lactose, dextrin, arabinose, rhamnose, xylose, raffinose, inulin, salicin, dulcitol, inositol, melezitose, adonitol.

Carbon utilization: utilizes glucose, lactose, sucrose, maltose, fructose, dextrin, mannose, arabinose, rhamnose, xylose, raffinose, inulin, mannitol, salicin, dulcitol, galactose, trehalose, inositol, cellobiose, melezitose, adonitol, glycogen, starch, chitin, asparaginate, succinate, malate, lactate, pyruvate $(0 \cdot 3 \%)$, acetate, $\beta$-alanine, glucosamine, ethanol, methanol, and tert.butanol. Does not utilize fumarate $(0.5 \%)$, malonate, tartrate, citrate, levulinate, propionate, salicylate, valerate, oxalate, butyrate, mandelate, benzoate $(0.5 \%), n$-amyl alcohol, iso-amyl alcohol, $n$-propanol, lumichrome.

Growth not inhibited by $10 \%$ sodium chloride.

Aerobic, facultative.

Optimum temperature: $\mathbf{2 0 - 3 0 ^ { \circ }}$. Grows at $4^{\circ}$.

Source: marine mud.

Habitat: sea water.

Achromobacter lipophagum n.sp. (from Greek, fat-eating)

Small rods : $0 \cdot 4$ by $1 \cdot 0 \mu$., occurring singly, in pairs, and small clumps, motile by means of peritrichous flagella. Gram-negative.

Agar colonies : circular, smooth, entire, glistening, opaque, and non-pigmented. Agar slant: abundant, filiform, glistening, opaque and non-pigmented.

Gelatin stab: saccate liquefaction.

Broth: uniform turbidity.

Litmus milk: becomes acid in 4 days with slight peptonization.

Hydrogen sulphide, indole nitrites, urease not formed.

Trimethylamine produced from trimethylamine oxide but not from choline and betaine.

Gelatin, casein, fats, starch, and chitin are hydrolysed.

Ammonia produced from peptone.

Produces acid but not gas from glucose, maltose and mannose. Does not ferment lactose, sucrose, fructose, dextrin, arabinose, rhamnose, xylose, raffinose, inulin, mannitol, sorbitol, salicin, dulcitol, trehalose, inositol, cellobiose, melezitose, adonitol.

Carbon utilization : utilizes glucose, lactose, sucrose, maltose, fructose, dextrin, mannose, arabinose, rhamnose, xylose, raffinose, inulin, mannitol, salicin, dulcitol, galactose, trehalose, inositol, cellobiose, melezitose, adonitol, glycogen, starch, chitin, asparaginate, succinate, malate, fumarate $(0 \cdot 5 \%)$, lactate, pyruvate $(0 \cdot 3 \%)$, acetate, $\beta$-alanine, glucosamine, ethanol, methanol, tert.-butanol. Does not utilize malonate, tartrate, citrate, 
levulinate, propionate, salicylate, valerate, oxalate, butyrate, mandelate, benzoate $(0.5 \%), n$-amyl alcohol, iso-amyl alcohol, $n$-propanol, lumichrome.

Growth not inhibited by $10 \%$ sodium chloride.

Aerobic, facultative.

Optimum temperature: 20-30 ${ }^{\circ}$. Grows at $4^{\circ}$.

Source: marine mud.

Habitat: sea water.

Five additional strains.

Achromobacter hyperopticum n.sp. (from Greek, hard to please)

Small rods: 0.2 by $0.6 \mu$, occurring singly, and in small clumps. Motile by means of peritrichous flagella, Gram-negative.

Agar colonies: small, punctiform, glistening, opaque and non-pigmented.

Agar slant: beaded, glistening, opaque and non-pigmented.

Gelatin stab: crateriform liquefaction.

Broth: moderate turbidity, surface ring adhering to the walls of the tube, and a slight stringy sediment.

Litmus milk: alkaline, with slight peptonization in 4 days.

Hydrogen sulphide, indole and urease not formed.

Nitrites are produced from nitrates.

Trimethylamine not produced from trimethylamine oxide, choline, or betaine. Gelatin, casein, fats, starch and chitin are hydrolysed.

Ammonia produced from peptone.

Produces acid but not gas from glucose, maltose, mannose, trehalose and cellobiose. Does not ferment lactose, sucrose, fructose, galactose, arabinose, dextrin, rhamnose, xylose, raffinose, inulin, mannitol, inositol, melezitose, adonitol.

Carbon utilization: utilizes glucose, sucrose, dextrin, mannose, raffinose, galactose, cellobiose, starch, glycogen, chitin, glucosamine, acetate. Does not utilize succinate, malate, fumarate $(0.5 \%)$, lactate, malonate, tartrate, citrate, pyruvate $(0 \cdot 3 \%)$, levulinate, $\beta$-alanine, asparaginate, propionate, salicylate, valerate, oxalate, butyrate, mandelate, benzoate $(\mathbf{0 . 5} \%)$, ethanol, methanol, iso-amyl alcohol, $n$-amyl alcohol, tert.-butanol, $n$-propanol, lumichrome.

Growth not inhibited by $10 \%$ sodium chloride.

Aerobic, facultative.

Optimum temperature: $20-30^{\circ}$. Grows at $4^{\circ}$.

Source: marine mud.

Habitat: sea water.

One additional strain.

Pseudomonas cryothasia n.sp (from Greek, cold enduring)

Small rods : $0 \cdot 3-0 \cdot 5$ by $0 \cdot 8-1 \cdot 2 \mu$, occurring singly, in pairs and small clumps. Motile by means of monotrichous flagellum.

Gram-negative. 
Agar colonies: circular, smooth, entire, convex, opaque, non-pigmented to pale cream.

Agar slant: filiform, glistening, opaque, non-pigmented to pale cream.

Gelatin stab: saccate to infundibuliform liquefaction.

Broth: moderate turbidity, slight surface scum, and slight flocculent sediment.

Litmus milk: acid with peptonization in 6 days.

Hydrogen sulphide, indole and urease not formed.

Nitrites are produced from nitrates.

Trimethylamine not produced from trimethylamine oxide, choline, or betaine.

Gelatin, casein, fats and chitin are hydrolysed; starch is not hydrolysed.

Acid or gas not produced from glucose, lactose, sucrose, maltose, fructose, dextrin, mannose, arabinose, rhamnose, xylose, raffinose, inulin, mannitol, salicin, dulcitol, galactose, trehalose, inositol, cellobiose, melezitose, adonitol.

Carbon utilization: utilizes glucose, maltose, dextrin, glycogen, pyruvate $(0.3 \%)$, acetate, asparaginate, succinate, malate, lactate, citrate, chitin, glucosamine. Does not utilize lactose, sucrose, fructose, mannose, arabinose, rhamnose, xylose, raffinose, inulin, mannitol, salicin, dulcitol, galactose, trehalose, inositol, cellobiose, melezitose, adonitol, fumarate $(0.5 \%)$, malonate, tartrate, levulinate, $\beta$-alanine, propionate, salicylate, valerate, oxalate, butyrate, mandelate, benzoate $(0.5 \%)$, starch, methanol, ethanol, $n$-amyl alcohol, iso-amyl alcohol, tert.-butanol, $n$-propanol, lumichrome.

Growth not inhibited by $10 \%$ sodium chloride.

Aerobic, facultative.

Optimum temperature: $20-30^{\circ}$. Grows at $4^{\circ}$.

Source: marine mud.

Habitat: sea water.

Two additional strains.

Pseudomonas subrubra n.sp. (from Latin, reddish)

Rods : $0 \cdot 5-0 \cdot 7$ by $1 \cdot 0-2 \cdot 2 \mu$, occurring singly, and in pairs. Motile with a single polar flagellum. Gram-negative.

Agar colonies : circular, smooth, entire, glistening, opaque, non-pigmented to pale pink.

Agar slant: filiform, smooth, glistening, opaque, non-pigmented to pale pink streak.

Gelatin stab: crateriform liquefaction.

Broth: moderate turbidity, fine granular sediment.

Litmus milk: acid with peptonization in $\mathbf{7 2} \mathrm{hr}$.

Hydrogen sulphide, indole and urease not formed.

Nitrites produced from nitrates.

Trimethylamine not produced from trimethylamine oxide, choline, or betaine.

Gelatin, casein, fats starch and chitin are hydrolysed.

Ammonia produced from peptone. 
Produces acid but no gas in glucose, maltose, fructose, dextrin, mannose, trehalose and cellobiose. Acid not produced in lactose, sucrose, arabinose, rhamnose, xylose, raffinose, inulin, mannitol, salicin, dulcitol, inositol, melezitose, adonitol.

Carbon utilization: growth on glucose, sucrose, maltose, fructose, dextrin, mannose, rhamnose, mannitol, galactose, trehalose, inositol, cellobiose, chitin, glycogen, starch, butyrate, valerate, asparaginate, glycine, succinate, malate, fumarate $(0.5 \%)$, lactate, acetate, citrate, pyruvate $(0.3 \%)$, levulinate, $\beta$-alanine, glucosamine, ethanol, methanol, tert.-butanol. No growth on lactose, arabinose, xylose, raffinose, inulin, salicin, dulcitol, melezitose, adonitol, malonate, tartrate, propionate, salicylate, oxalate, mandelate, benzoate $(0.5 \%), n$-amyl alcohol, iso-amyl alcohol, $n$-propanol and lumichrome.

Growth not inhibited by $10 \%$ sodium chloride.

Aerobic, facultative.

Optimum temperature: $20-30^{\circ}$. Grows at $4^{\circ}$.

Source: marine mud.

Habitat: sea water.

One additional strain.

Flavobacterium indoltheticum n.sp. (from Greek, indole making)

Rods: $0.4-0.9$ by $1.0-1.5 \mu$, occurring singly, in pairs and occasional clumps. Motile by means of peritrichous flagella. Gram-negative.

Agar colonies : circular, smooth, entire, opaque, yellow to yellow-orange pigment.

Agar slant: yellow to yellow-orange streak, smooth, opaque.

Gelatin stab: crateriform liquefaction.

Broth: turbid, slight surface scum, yellow to orange sediment.

Litmus milk: acid in $\mathbf{4 8} \mathrm{hr}$.

Hydrogen sulphide, nitrites and urease not formed.

Indole is formed.

Trimethylamine not produced from trimethylamine oxide, choline or betaine. Gelatin, casein, starch and chitin are hydrolysed; fats not hydrolysed.

Ammonia produced from peptone.

Acid but not gas produced in glucose, sucrose, maltose, fructose, dextrin, mannose, raffinose, trehalose and cellobiose. Acid not produced in lactose, arabinose, rhamnose, xylose, inulin, mannitol, salicin, dulcitol, inositol, melezitose, adonitol.

Carbon utilization: growth on glucose, sucrose, maltose, fructose, dextrin, mannose, raffinose, salicin, cellobiose, trehalose, pyruvate $(0 \cdot 3 \%)$, acetate, chitin, starch, glycogen and glucosamine. No growth on lactose, arabinose, rhamnose, xylose, inulin, mannitol, dulcitol, galactose, inositol, melezitose, adonitol, succinate, malate, fumarate $(0.5 \%)$, lactate, malonate, tartrate, citrate, levulinate, $\beta$-alanine, asparaginate, propionate, salicylate, valerate, oxalate, butyrate, mandelate, benzoate (0.5\%), ethanol, methanol, $n$-amyl alcohol, iso-amyl alcohol, tert.-butanol, $n$-propanol, lumichrome. 
Growth is inhibited by $10 \%$ sodium chloride.

Aerobic, facultative.

Optimum temperature: $20-30^{\circ}$. Does not grow at $4^{\circ}$.

Source: marine mud.

Habitat: sea water.

One additional strain.

Micrococcus colpogenes n.sp. (from Greek, gulf-born)

Cocci, occurring in clumps and occasional pairs. Non-motile, Gram-positive. Agar colonies: circular, smooth, entire, raised, glistening, yellow.

Agar slant: filiform, glistening, yellow streak.

Gelatin stab: no liquefaction.

Broth: moderate turbidity, with slight granular sediment.

Litmus milk: unchanged.

Hydrogen sulphide and indole not formed.

Nitrites and urease are formed.

Trimethylamine not produced from trimethylamine oxide, choline, or betaine. Gelatin, casein, fats and starch are not hydrolysed; chitin is hydrolysed.

Acid or gas not produced from glucose, lactose, sucrose, maltose, fructose, dextrin, mannose, arabinose, rhamnose, xylose, raffinose, inulin, mannitol, salicin, dulcitol, galactose, trehalose, inositol, cellobiose, melezitose, adonitol.

Carbon utilization: utilizes glucose, maltose, mannitol, malate, pyruvate $(0 \cdot 3 \%)$, acetate, succinate, glycogen, chitin, glucosamine.

Does not utilize fumarate $(0.5 \%)$, lactate, malonate, tartrate, citrate, levulinate, $\beta$-alanine, asparaginate, propionate, salicylate, valerate, oxalate, butyrate, mandelate, benzoate $(0.5 \%)$, starch, ethanol, methanol, $n$-amyl alcohol, iso-amyl alcohol, tert.-butanol, $n$-propanol, lumichrome.

Growth is inhibited by $10 \%$ sodium chloride but not by $4 \%$.

Aerobic, facultative.

Optimum temperature: 20-30 ${ }^{\circ}$. Does not grow at $4^{\circ}$.

Source: marine mud.

Habitat: sea water.

One additional strain.

\section{REFERENCES}

BENECKE, W. (1905). U'ber Bacillus chitinovorus, einen chitinzersetzenden Spaltpilz. Bot. Ztg. (1. Abt.) 63, 227.

Benton, A. G. (1935). Chitinovorous bacteria. A preliminary survey. J. Bact. 29, 449.

Bucherer, H. (1935). Úber den mikrobiellen Chitinabbau. Zbl. Bakt. (2. Abt.) 93, 12.

Dehn, M. von (1937). Zur Frage der Natur der peritrophischen Membran bei den Insekten. Z. Zellforsch. 25, 787.

Elson, L. A. \& Morgan, W. T. J. (1933). A colorimetric method for the determination of glucosamine and chondrosamine. Biochem. $J .27,1824$.

Erikson, D. (1941). Studies on some lake-mud strains of Micromonospora. J. Bact. 41, 277. 
Folpmers, T. (1921). Die Zersetzung des Chitins und des Spaltungsproduktes desselben, des Glucosamins, durch Bakterien. Chem. Weekbl. 18, 249.

Gurck, D. (1949). Techniques of Histo- and Cytochemistry, p. 52. New York: Interscience Publishers.

Hock, C. W. (1941). Marine chitin-decomposing bacteria. J. mar. Res. 4, 99.

Johnson, D. E. (1932). Some observations of chitin-destroying bacteria. J. Bact. 24, 335.

NABel, K. (1939). Über die Membran niederer Pilze, besonders von Rhizidiomyces bivellatus nov. spec. Arch. Mikrobiol. 10,515. (Abstract from Biol. Abstr. 1941, 15, 9118.)

Rammelberg, G. (1931). Beitrag zur Kenntnis des Chitins der Pilze und Krabben. Bot. Arch. 32, 1.

Rittenberg, S. C., Anderson, D. Q. \& ZoBell, C. E. (1937). Studies on the enumeration of marine anaerobic bacteria. Proc. Soc. exp. Biol., N.Y., 35, 652.

Skinner, C. E. \& Dravis, F. (1937). A quantitative determination of chitindestroying micro-organisms in soil. Ecology, 18, 391.

Stanier, R. Y. (1947). Studies on nonfruiting myxobacteria. I. Cytophaga johnsonnae, n.sp. A chitin-decomposing myxobacterium. J. Bact. 53, 297.

Steiner, M. (1931). Beiträge zur Kenntnis der Zellulose und des Chitinabbaues durch Mikroorganismen in stehenden Binnengewässern. 75 Jahre Stelle Matutina, Festschrift, Feldkirch. 2, 367. (Abstract from Biol. Abstr. 1934, 8, 2815.)

Stuart, L. S. (1936). A note on halophilic chitinovorus bacteria. J. Amer. Leath. Chem. Ass. 31, 119. (Cited by ZoBell, 1946.)

WAKsMAN, S. A. (1933). On the distribution of organic matter in the sea bottom and the chemical nature and origin of marine humus. Soil Sci. 36, 125.

Waksman, S. A., Carex, C. L. \& Reuszer, H. W. (1933). Marine bacteria and their role in the cycle of life in the sea. I. Decomposition of marine plant and animal residues by bacteria. Biol. Bull. Woods Hole, 65, 57 .

ZoBeld, C. E. (1946). Marine Microbiology, p. 143. Waltham, Mass.: Chronica Botanica Co.

ZoBell, C. E. \& ANDERson, D. Q. (1936). Vertical distribution of bacteria in marine sediments. Bull. Amer. Ass. Petrol. Geol. $20,258$.

ZoBell, C. E. \& Rittenberg, S. C. (1938). The occurrence and characteristics of chitinoclastic bacteria in the sea. J. Bact. 35, 275.

(Received 2 April 1951) 